\title{
Physical fitness in institutionalized older adults with dementia: association with cognition, functional capacity and quality of life
}

\author{
A. Sampaio ${ }^{1}$ - I. Marques-Aleixo ${ }^{1,2} \cdot$ A. Seabra ${ }^{1} \cdot$ J. Mota $^{1} \cdot$ E. Marques $^{3} \cdot$ J. Carvalho $^{1}$
}

Received: 30 August 2019 / Accepted: 11 December 2019 / Published online: 11 January 2020

(c) The Author(s) 2020

\begin{abstract}
This cross-sectional study investigated the association of physical fitness with cognitive function, functional capacity and quality of life among institutionalized older adults with dementia. One hundred and two older adults aged $78.0 \pm 8.4$ years, predominantly female (67.6\%), with neurocognitive disorder due to Alzheimer's disease (AD) (49.2\%), vascular dementia (14.7\%), Parkinson's disease (2\%), dementia with Lewy bodies (2\%) or unspecified dementia (32.1\%) participated in the present study. Regression analyses were used to examine associations between physical fitness components (Senior Fitness Test) and cognitive function (Mini-Mental State Examination), functional capacity (Katz Index of Independence in Activities of Daily Living) and Quality of Life (QoL)-Alzheimer's Disease scale. Univariate regression indicates that strength, flexibility, agility/dynamic balance and aerobic endurance are relevant for cognitive function, physical capacity and perceived QoL in institutionalized older people with dementia. After multiple regression analyses, adjusted for body mass index (BMI), results showed that aerobic endurance had a significant positive association with Total Katz Index. For both, caregiver perception of QoL-AD and global QoL-AD, BMI remained significantly and positively associated. Agility-dynamic balance presented a significant negative relation with global QoL-AD. Overall, our findings suggest that better physical fitness is important for cognition and autonomous functional capacity and that it has positive repercussions on the QoL in institutionalized older adults with dementia. Consequently, exercise-based therapeutic strategies aiming to improve physical fitness should be implemented.
\end{abstract}

Keywords Alzheimer's disease $\cdot$ Physical fitness $\cdot$ ADL $\cdot$ Ageing $\cdot$ Institutionalization

\section{Introduction}

Dementia is a progressive degenerative syndrome that affects memory, thinking, behaviour and the ability to perform activities of daily life (ADL) [1]. According to the Fifth Edition of the American Psychiatric Association's Diagnostic and Statistical Manual (DSM-5), dementia is a major

A. Sampaio

arnaldina@gmail.com

1 CIAFEL-Research Center in Physical Activity, Health and Leisure, Faculty of Sport, University of Porto, Rua Dr. Plácido Costa 91, 4200-450 Porto, Portugal

2 Faculty of Psychology, Education and Sports, Lusófona University of Porto, Rua de Augusto Rosa 24, 4000-098 Porto, Portugal

3 Research Center in Sports Sciences, Health Sciences and Human Development, CIDESD, University Institute of Maia, ISMAI, Maia, Portugal neurocognitive disorder (MND) and is caused by a variety of neurodegenerative diseases, including Alzheimer's disease ( $\mathrm{AD}$, most common type), vascular dementia, dementia with Lewy bodies and Parkinson's disease. Dementia affects over 48 million people worldwide, and it has been predicted that this number will double every 20 years [2]. Due to its progressive degenerative nature, this syndrome contributes to physical, cognitive and social disabilities that culminate in functional dependency, usually leading to institutionalization. Therefore, dementia has devastating social consequences, not least on healthcare costs, effectively making it one of the leading age-related health problems affecting worldwide [1]. Major international public health organizations and national governments are calling for policies to solve and/or mitigate the global burden of dementia [3]. Among the innumerous preventive and therapeutic strategies used to counteract dementia-associated diseases, physical exercise has been considered a promising non-pharmacological approach. 
Ageing is associated with a decrease in all health-related components of physical fitness, including cardiorespiratory endurance, muscular endurance, muscular strength, body composition and flexibility [4]. Moreover, as older adults become less physically active during the ageing process $[5$, 6], their physical fitness is negatively affected. Physical fitness components are always required for carrying out ADL, such as getting up from a sitting or lying position, taking a shower, avoiding obstacles and walking [5, 7]. However, functional capacity, in the sense of the ability to perform ADL autonomously [8], is not only associated with physical fitness but also with psychological factors acting together over extended time [9]. Frequently, older adults with dementia live in nursing homes and other institutional settings, which lack opportunities for physical activity [10], thus exacerbating the decline of physical fitness.

Conversely, it seems that higher levels of physical activity/fitness could help reduce the incidence of dementia. In a large cohort of men and women surveyed over 17 years, having better fitness was associated with a lower risk of mortality from dementia [11]. A prospective study with 163,000 older adults found that physical activity can reduce the risk of dementia by $28 \%$ and $\mathrm{AD}$ by $45 \%$ [12].

Exercise interventions for people with dementia have shown positive results in the ability to perform ADLs. Based on randomized controlled trials, Forbes and collaborators [13] concluded that older adults with dementia did find exercise beneficial to their ability to perform ADL. A recent meta-analysis with ten randomized controlled trials showed a moderate-to-large positive effect after combined cognitive-physical interventions for ADL [14].

The existing body of literature reveals modest findings between fitness training and improvements in cognition. Karssemeijer and collaborators [14] reported a small-tomedium positive effect of combined cognitive-physical interventions on global cognitive function in older adults with mild cognitive impairment (MCI) or dementia. Another meta-analysis that included 39 studies has shown that physical exercise improved cognitive function in the over $50 \mathrm{~s}$, regardless of the cognitive status of participants evidence [15]. However, a previous Cochrane review that examined the effect of exercise for older people with dementia revealed no clear evidence of benefit from exercise on cognitive functioning [13]. Therefore, the association between participation in physical activity programs and cognition in older adults with dementia still needs to be further investigated.

Quality of life (QoL) is a multidimensional concept, and physical fitness has been considered one of its critical components due to its importance in performing ADL independently [16]. In fact, among older adults with dementia, a negative QoL is associated with diminished physical and cognitive function [17]. As a factor that can be modified by increasing physical activity, physical fitness is essential for maintaining the motor skills that are critical for performing $\mathrm{ADL}$ and consequently for enhancing QoL in people with dementia [13].

Although there are a growing number of studies on exercise programs applied to adults with dementia suggesting beneficial effects, a wide variety of methodologies regarding duration (e.g. 2 weeks; 18 months), frequency (e.g. twice per week; daily) and exercise type (e.g. aerobic, strength, balance) and different dementia population (stages of dementia; institutionalized or in community settings, among others) may contribute to inconsistent or even contradictory outcomes [13]. Therefore, further research is needed to develop efficient exercise programs, and it is also necessary to identify which physical fitness components are more relevant for institutionalized older adults with dementia. This study aimed to clarify the association between different physical fitness components, as a modifiable factor throughout life particularly with exercise engagement, with cognitive function, functional capacity and QoL in institutionalized older people with dementia.

\section{Materials and methods}

\section{Participants and study design}

The participants of this cross-sectional study were recruited in six different Portuguese nursing homes. One hundred and two older adults from both genders, aged 65-93 years and all diagnosed by a physician with an age-related neurocognitive disorder, agreed to participate in the study. The eligible subject pool was restricted to older adults with the following characteristics: age $\geq 65$ years, institutionalized for more than 6 months, diagnosis of an age-related neurocognitive disorder and absence of any diagnosed or self-reported musculoskeletal or cardiovascular disorders that would contraindicate participation in physical fitness testing. During the initial screening visit, all participants, formal caregivers and institutions received a complete explanation of the purpose, risks and procedures of the study. Written informed consent was provided, and the review boards of the six nursing homes approved all methods and procedures. The investigation was in full compliance with the Helsinki Declaration [18]. The sociodemographic and clinical characteristics of participants at baseline are shown in Table 1.

\section{Measurements}

All measurements were performed by the same evaluators, in six different nursing homes. At each nursing home, the test was of 1-week duration. In the morning, participants completed anthropometric measurements (weight and height) and performed Senior Fitness Test (SFT) battery and Quality 
of Life-Alzheimer's Disease scale (QoL-AD) questionnaire, while, in the afternoon, formal caregivers filled Katz Index and QoL-AD questionnaire. Despite different institutions, all assessments were organized in the same way.

\section{Physical fitness}

Physical fitness was assessed with the SFT [7], which is considered a reliable instrument for assessing physical fitness in older adults ( $\geq 60$ years of age), including older people with cognitive impairment [19]. Participants performed six tests: chair stand test (to assess lower body strength); arm curl test (to measure upper body strength); 2-minute step test (to assess aerobic endurance); chair sit and reach test (to assess lower body flexibility); back

Table1 Characteristics of the participants

\begin{tabular}{ll}
\hline & Participants $(n=102)$ \\
\hline Age (years) & $78.0 \pm 8.4$ \\
Men, no. (\%) & $33(32.4 \%)$ \\
Educational level (years) & $3.48 \pm 3.3$ \\
Neurocognitive disorder due to, no. (\%) & \\
Alzheimer's disease & $50(49.2 \%)$ \\
Vascular disease & $15(14.7 \%)$ \\
Parkinson's disease & $2(2 \%)$ \\
Lewy bodies disease & $2(2 \%)$ \\
Unspecified & $33(32.1 \%)$ \\
Diagnosis (others than NCD), no. (\%) & \\
Hypertension & $32(31.4 \%)$ \\
Heart disease & $20(19.6 \%)$ \\
Diabetes mellitus & $15(14.7 \%)$ \\
Osteoporosis & $9(8.8 \%)$ \\
Blood pressure, (mmHg) & \\
Systolic & $125.8 \pm 20.8$ \\
Diastolic & $73.9 \pm 12.3$ \\
Physical fitness & \\
Lower body strength (Rps) & $25.3 \pm 4.6$ \\
Upper body strength (Rps) & \\
Lower body flexibility [25] & $10.7 \pm 5.0$ \\
Agility/dynamic balance (sec) & $19.2 \pm 10.8$ \\
Upper body flexibility [25] & $19.2 \pm 10.5$ \\
Aerobic endurance (step) & $40.4 \pm 14.2$ \\
MMSE (points) & $64.5 \pm 31.9$ \\
Total Katz (points) & $17.9 \pm 4.9$ \\
Quality of life (points) & $13.5 \pm 4.2$ \\
Participant total score & \\
Caregiver total score & $25.8 \pm 5.2$ \\
Global total score & $24.2 \pm 5.6$ \\
\hline
\end{tabular}

Number and proportional distributions are presented for categorical variables: gender; neurocognitive disorders and diagnosis. All other variables are mean \pm standard deviation scratch test (to assess upper body flexibility); and 8-foot up and go test (to assess agility and dynamic balance).

\section{Cognitive function}

The Mini-Mental State Examination (MMSE) [20] was used for a global cognitive evaluation. This instrument is clinically used to assess cognitive mental status, as well as to detect and follow the course of mental illness. It assesses orientation, attention, immediate and short-term recall, language and the ability to follow simple verbal and written instructions. A total score categorizes the individual on a scale of cognitive function ranging from 0 to 30 [20]. MMSE normative values consider the subjects' educational level. Operational cut-off values for the Portuguese population [21] are 22 (for 0-2 years of literacy), 24 (for 3-6 years of literacy) and 27 (for more than 6 years of literacy).

\section{Functional capacity}

A Portuguese modified version of Katz Index [22] was used to evaluate participant's functioning capacity based on caregiver report. The index includes six items: bathing, dressing, transferring, feeding, incontinence, toileting and the sum of all items to calculate the Katz total [22, 23]. Independence levels for the ADL questions are recorded on a scale of $0-4$, divided in the following categories: 1 dependent; 2 independent with help; 3 independent with supervision; 4 independent [22].

\section{Quality of life}

The QoL-AD scale [24] was used to measure the participant's QoL. The questionnaire includes 13 items: physical health, energy, mood, living situation, memory, family, marriage, friends, self as a whole, ability to do chores, ability to do things for fun, financial situation and QoL as a whole. The QoL-AD uses both self-report and the caregiver's reports of the participant's QoL and is scored on a 4-point Likert scale ranging from 1 (poor) to 4 (excellent), with total scores ranging between 13 and 52 points.

\section{Statistical analysis}

Characteristics of the sample were expressed either as means and standard deviations or proportions. Multiple regression analyses with cognition, functional capacity and QoL as the dependent variables and physical fitness components as independent variables were used. Candidate variables for the 
multivariable model were screened with univariate methods. At each step, the independent variable not in the model that had the smallest p-value was entered, and variables already in the model were removed if their $p$ value became larger than the significance level. The model was terminated when no more variables were eligible for inclusion or removal. Significance level in all analyses was set at 0.05 . SPSS version 24.0 was used in all analyses.

\section{Results}

The characteristics of the participants are summarized in Table 1. The 102 participants were predominantly female $(67.6 \%)$ and had a neurocognitive disorder (NCD) due to AD. Hypertension, a minor heart condition, diabetes mellitus and osteoporosis were the other main diagnoses besides NCD.

The association between the cognition (assessed by total MMSE) and physical fitness components is presented in Fig. 1. Except for the lower body strength and flexibility $(p>0.05)$, physical fitness components were significantly associated with cognition. Aerobic endurance and upper body strength were the components with a higher relation with cognition (Fig. 1b, f), explaining $\approx 7.4 \%$ and $7.2 \%$ of the variance, respectively.

The association between functional capacity (assessed by total Katz) and physical fitness components is presented in Fig. 2. Lower body flexibility and agility and dynamic balance were not associated with cognition $(p>0.05)$. All the other physical fitness components were significantly associated with functional capacity. Aerobic capacity was the component with the higher relation with functional capacity (Fig. 2f), explaining $\approx 12.5 \%$ of the variance.

The association between QoL perceived by the participant and physical fitness components is presented in Fig. 3. Flexibility (upper and lower body) was not significantly associated with QoL $(p>0.05)$. All the other physical fitness components were significantly associated with QoL perceived by the participant. Aerobic endurance and agility/dynamic balance were the components with the higher relation with QoL perceived by the participant (Fig. 4f, d), explaining $\approx$ $5.9 \%$ and $5.8 \%$ of the variance, respectively.

The association between QoL perceived by the caregiver and physical fitness components is presented in Fig. 4. Flexibility (upper and lower body) was not associated with QoL perceived by the caregiver $(p>0.05)$. All the other physical fitness components were significantly associated with QoL perceived by the participant. Lower and upper body strength was the components with the higher relation with QoL perceived by the participant (Fig. 4a, b), explaining $\approx 12 \%$ and $14 \%$ of the variance, respectively.

The association between global QoL and physical fitness components is presented in Fig. 5. Flexibility (upper and lower body) was not associated with QoL perceived by the caregiver $(p>0.05)$. All the other physical fitness components were significantly associated with QoL perceived by the participant. Lower body strength and aerobic endurance were the components with a higher relation a

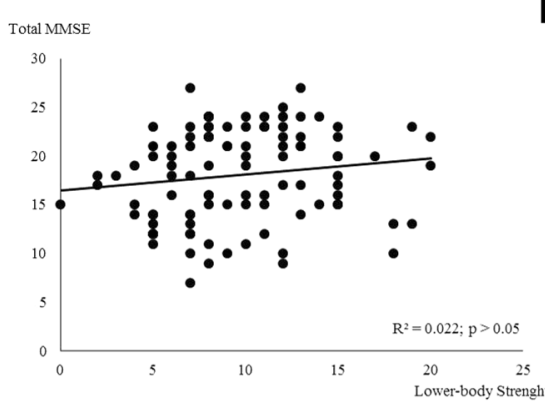

d

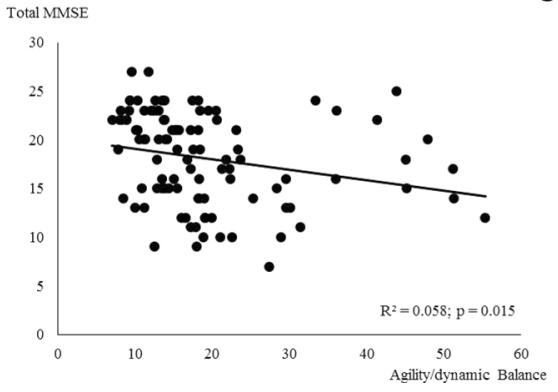

b
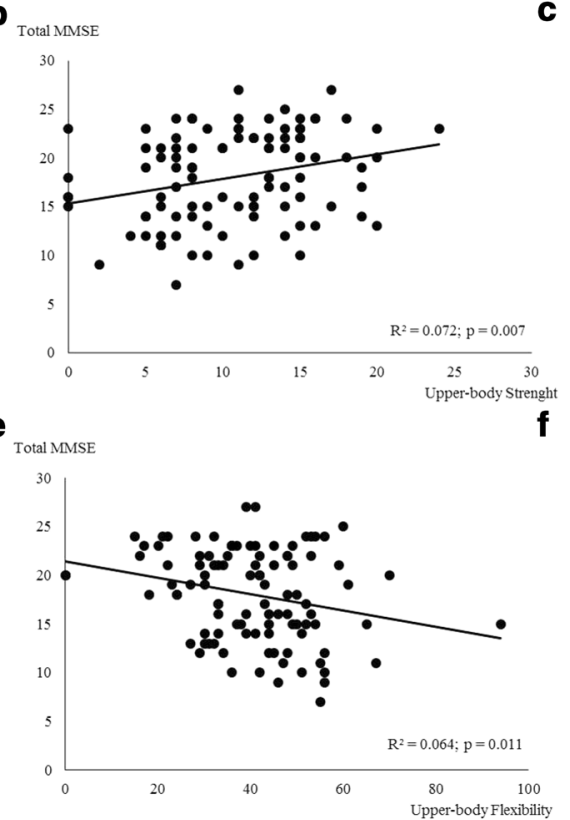

c
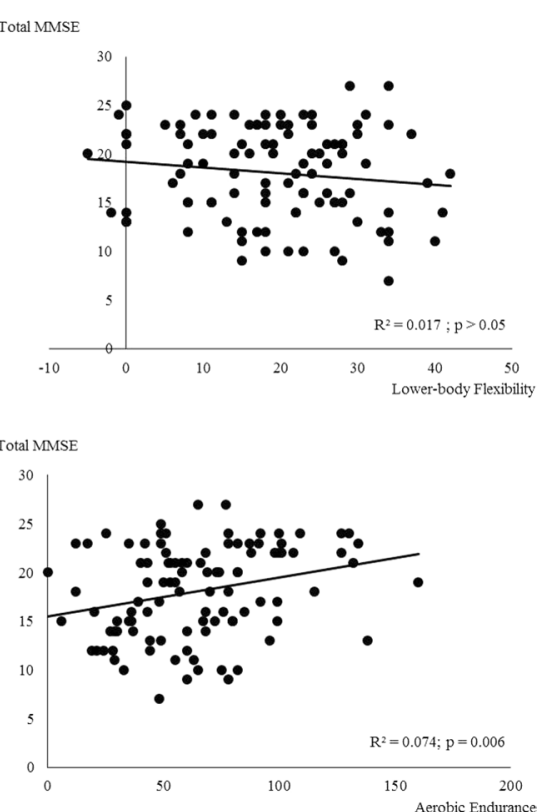

Fig. 1 Regression relationships between Total MMSE score (points) and physical fitness components 

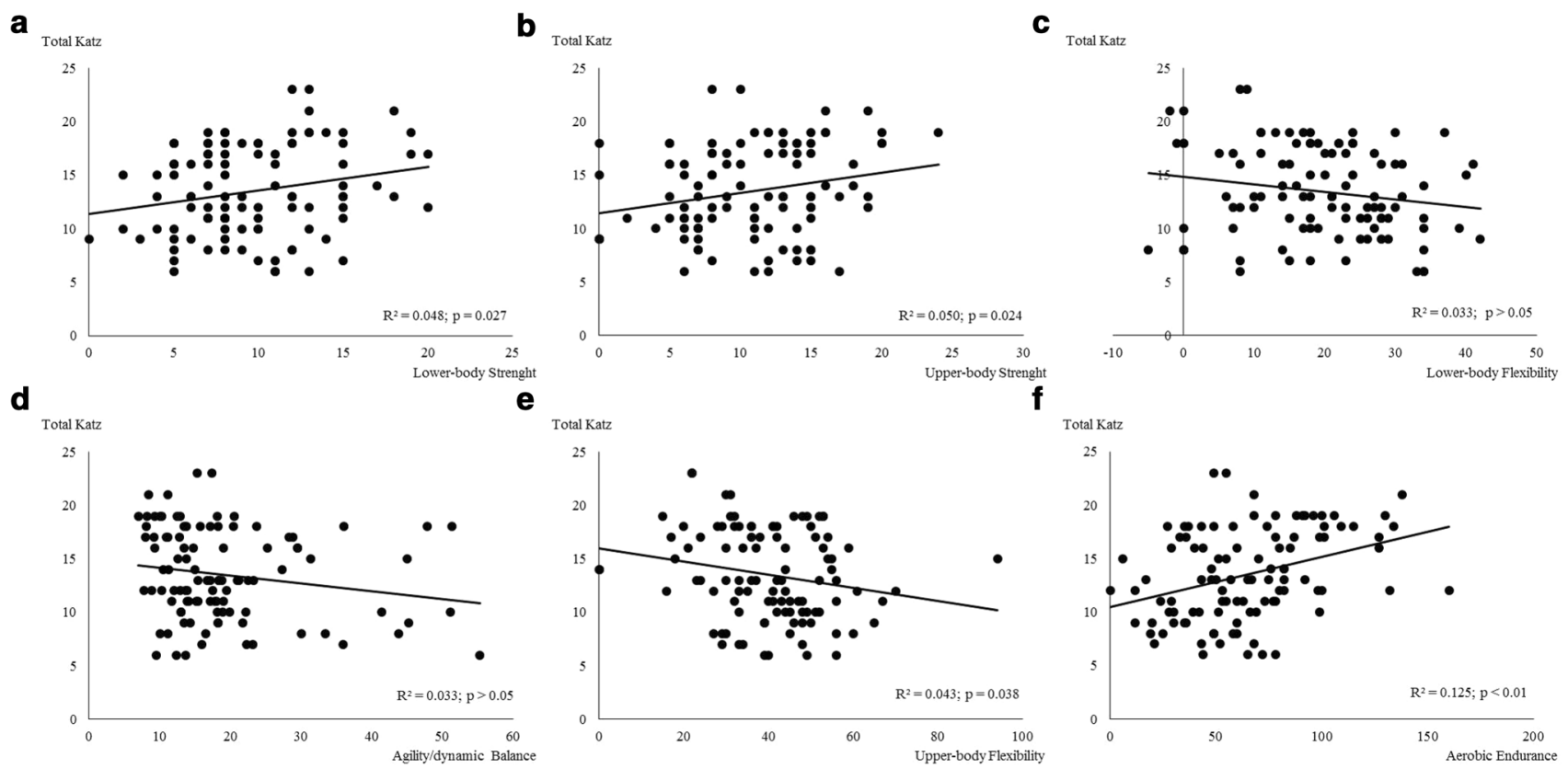

Fig. 2 Regression relationships between functional capacity (total Katz, points) and physical fitness components

a

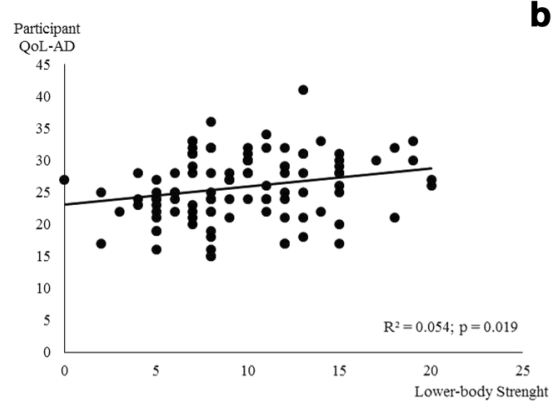

d

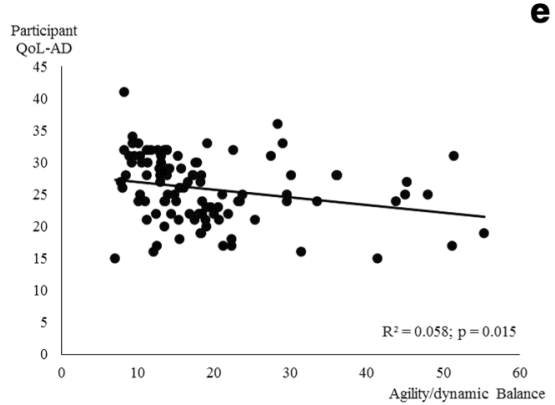

b

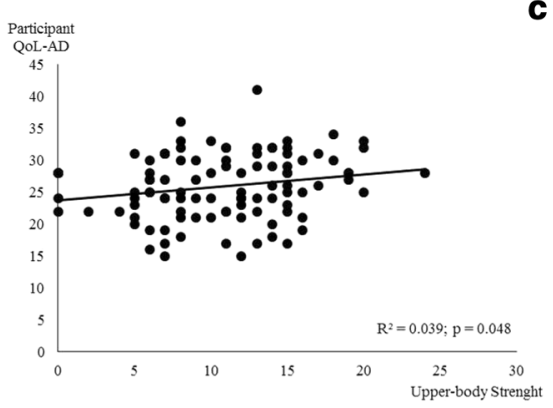

e

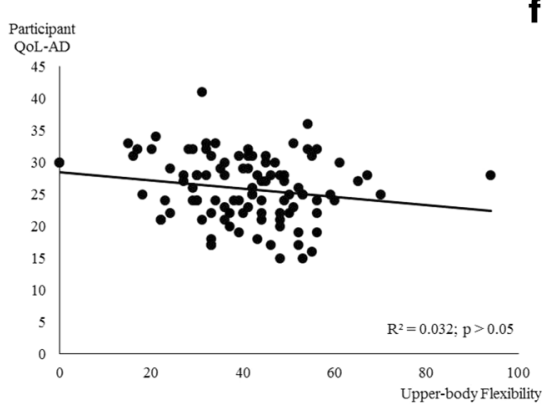

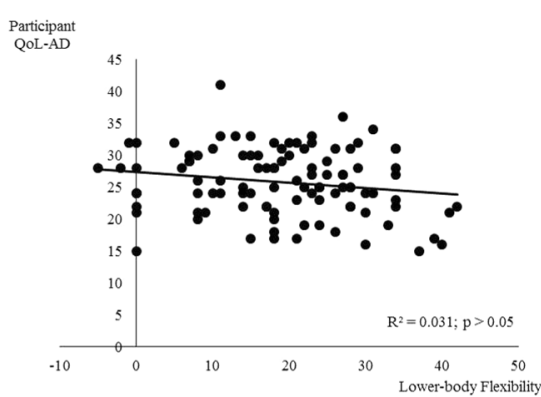

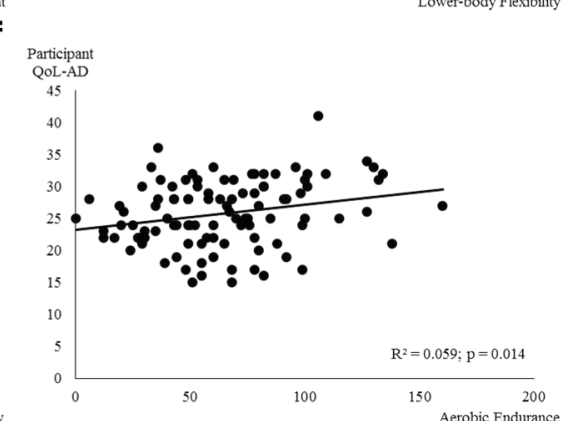

Fig. 3 Regression relationships between quality of life perceived by the participant (participant QoL-AD, points) and physical fitness components

with global QoL (Fig. 5a, f), explaining $\approx 9.7 \%$ of the variance for both components.

Adjusted coefficients and 95\% CI with cognition, functional capacity and quality of life as the dependent variables in linear multiple regression analyses are presented in Table 2. Age, gender and educational level were not correlated with cognition, functional capacity or QoL (data not shown); thus, they were not included as confounding variables in the regression models. As BMI was associated with global QoL and caregiver's perception of QoL (data not shown), this variable was included as confounders variable in the multiple regression models for global QoL 

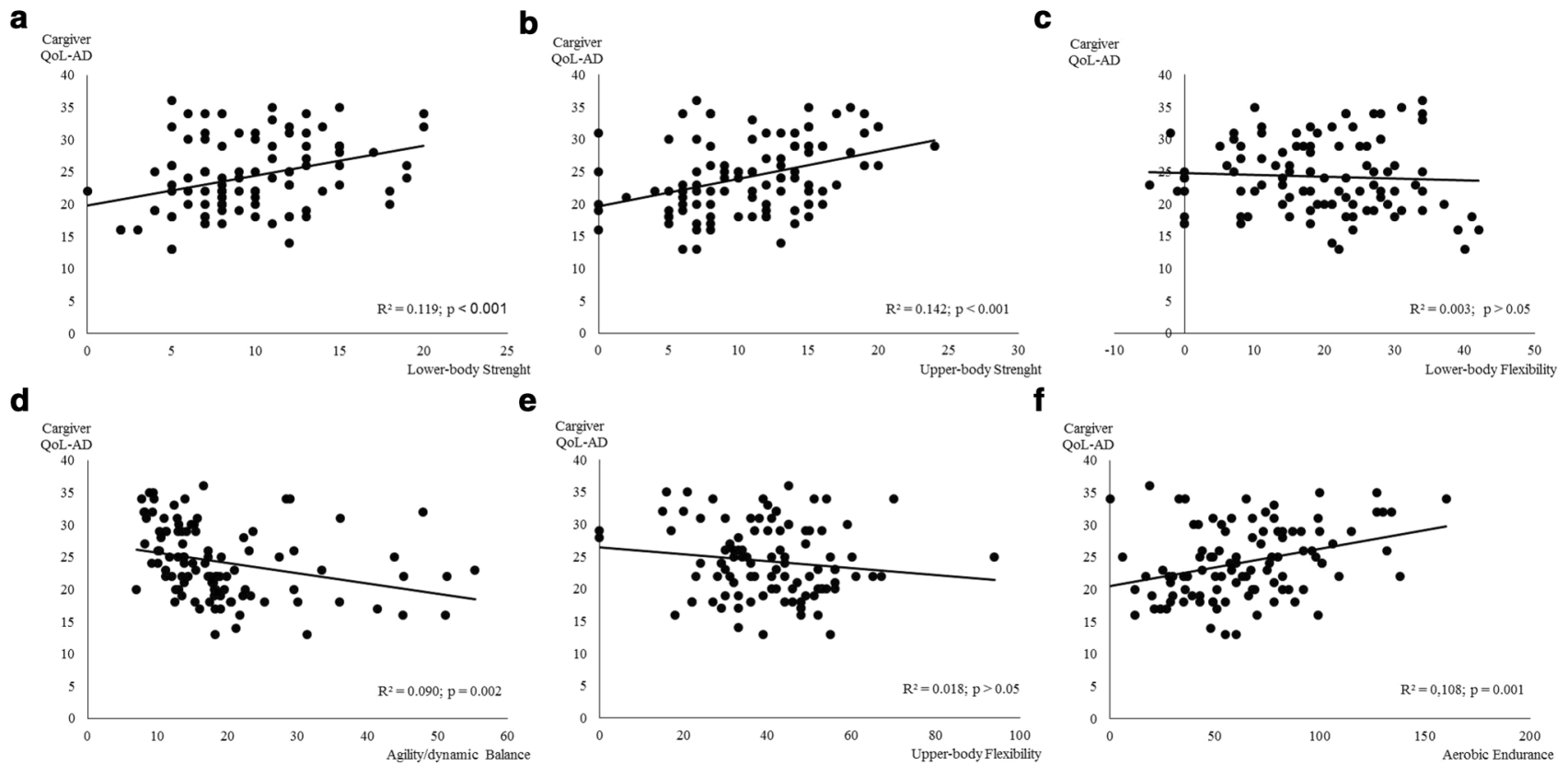

Fig. 4 Regression relationships between the quality of life of the participant perceived by the caregiver (Caregiver QoL-AD, points) and physical fitness components
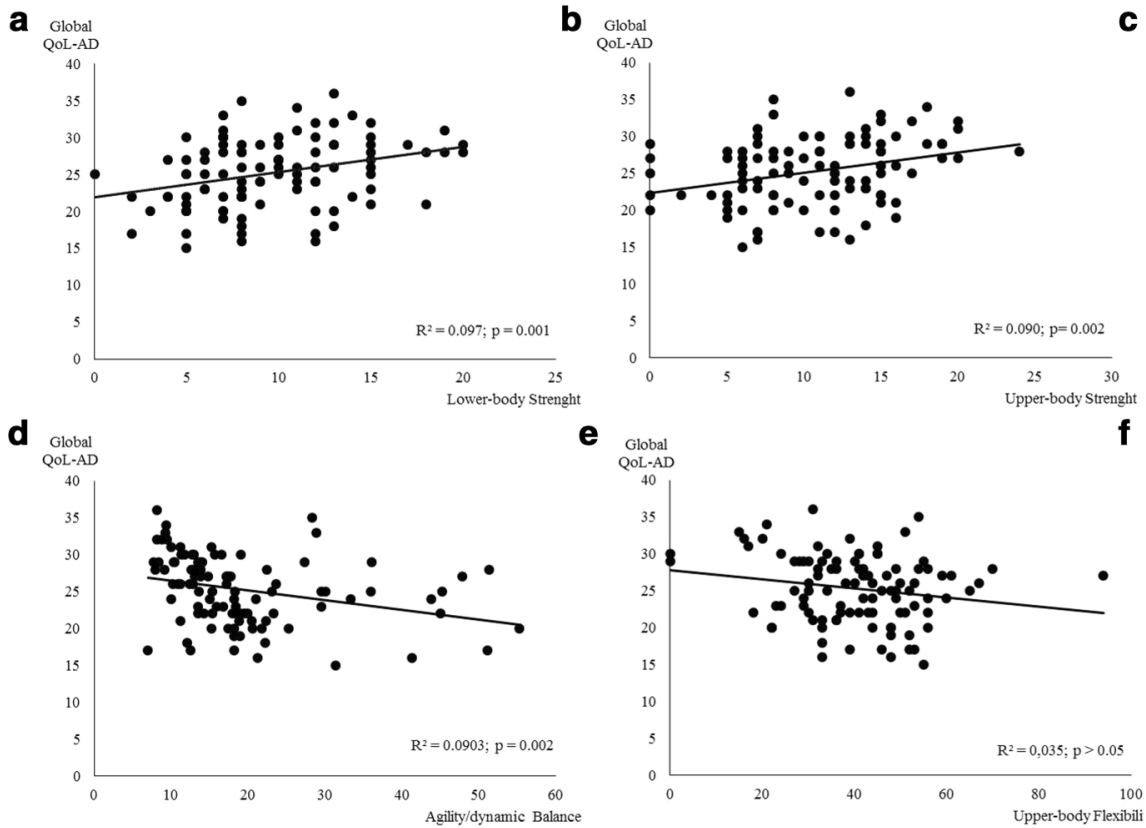

Global
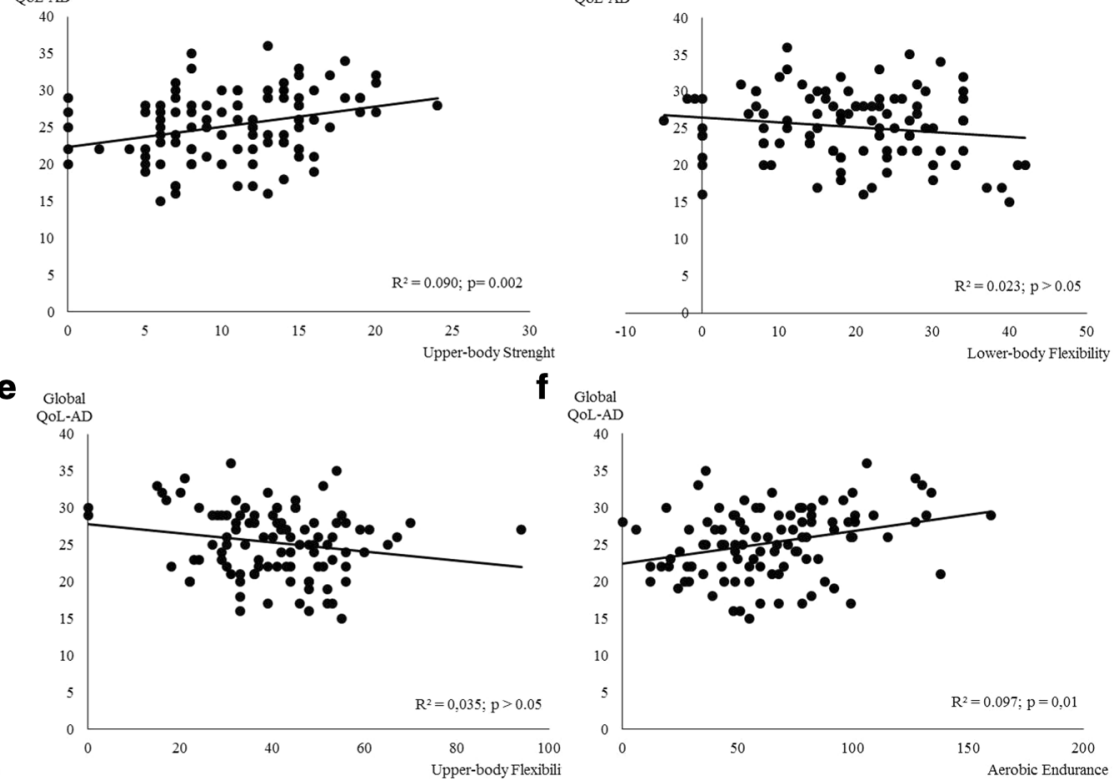

Fig. 5 Regression relationships between global quality of life of the participant (perception of participant + perception of the caregiver) (global QoL-AD, points) and physical fitness components

and caregiver's perception of QoL. Aerobic endurance had a significant positive association with total Katz. For both caregiver perceptions of QoL-AD and global QoL-AD, BMI remained significantly and positively associated. Agility-dynamic balance presented a significant negative relation with global QoL-AD. Additionally, lower body strength had a significant positive effect on caregiver QoL-AD. Neither component of physical fitness was associated with the variables cognition nor was participant perception of $\mathrm{QoL}$, 
Table 2 Multiple linear regression analyses of associations between the cognition, functional capacity and quality of life and the components of physical fitness

\begin{tabular}{llcc}
\hline $\begin{array}{l}\text { Multiple linear } \\
\text { regression }\end{array}$ & Adjusted $R^{2}$ & Adjusted $\beta(95 \%$ CI $)$ & $p$ value \\
\hline $\begin{array}{l}\text { Cognition } \\
\text { a }\end{array}$ & & & \\
$\begin{array}{c}\text { Functional capacity } \\
\text { Aerobic endurance }\end{array}$ & 0.097 & $0.04(0.01-0.08)$ & 0.012 \\
$\begin{array}{l}\text { Participant QoL-AD } \\
\text { Caregiver QoL-AD }\end{array}$ & & & \\
$\begin{array}{l}\text { BMI } \\
\text { Lower body strength }\end{array}$ & 0.346 & $0.42(0.15-0.70)$ & 0.003 \\
$\begin{array}{l}\text { Global QoL-AD } \\
\text { BMI }\end{array}$ & & $0.51(0.03-0.98)$ & 0.038 \\
Agility/dynamic & 0.295 & $0.31(0.09-0.54)$ & 0.008 \\
$\quad$ balance & & $-0.18(-0.30-0.06)$ & 0.003 \\
\hline
\end{tabular}

${ }^{a}$ Multiple regression adjusted for upper body strength, agility/ dynamic balance, upper body flexibility and aerobic endurance

${ }^{b}$ Multiple regression adjusted for lower and upper body strength, upper body flexibility and aerobic endurance

${ }^{\mathrm{c}}$ Multiple regression adjusted for lower and upper body strength, agility/dynamic balance and aerobic endurance

${ }^{\mathrm{d}}$ Multiple regression adjusted for lower and upper body strength, agility/dynamic balance, aerobic endurance and BMI

suggesting that for these variables, there is no physical fitness component that stood out above the others.

\section{Discussion}

Although the relationship between higher levels of physical activity and functional capacity has been established previously [25, 26], the association between the specific dimensions of the physical fitness and the ability to perform ADL are still unclear, particularly among older adults with dementia. Moreover, studies regarding the impact of physical fitness on cognition mainly investigate aerobic fitness [13] while the association between different components of physical fitness and cognitive function needs to be clarified in this population. Overall, this study demonstrates that different components of physical fitness are relevant for cognitive function and functional capacity in institutionalized older people with dementia. As physical fitness can modulate the cognitive function and functional capacity and as these are important for QoL [13], this study also highlights the association between different physical fitness components and the QoL in this particular population.

\section{Strength}

The prevalence of age-related loss of muscle mass and strength (sarcopenia) seems to be more predominant in older adults with dementia than older adults without dementia [27]. Gillette-Guyonne et al. [28] reported a sarcopenia prevalence of $40.6 \%$ in female subjects with $\mathrm{AD}$ in comparison with $21.9 \%$ in age-matched controls with no AD. Although the mechanisms behind these differences are not completely clear, dementia and sarcopenia may share certain mechanisms of pathogenesis [27, 29], such as malnutrition, hormonal changes, oxidative stress, inflammatory process and usually involving a decreased exercise and activity [30]. Previous reports suggested that the unintended weight loss and decreased BMI [31] observed in older adults with AD were predominantly related to the loss of lean mass (i.e. sarcopenia) [32]. In our study, multivariate linear regression showed that BMI was positively associated with caregivers' perception and with global QoL, and thus, it is possible to speculate that the amount of lean mass influences the QoL in older adults with dementia. Sarcopenia has been suggested to mediate the association between poor cognition and functional decline in cognitively impaired older adults [29]. Nourhashemi [33] and collaborators found that low cognitive function was associated with muscle loss in a group of over 7,000 community-dwelling older women. Raji [34] and collaborators showed an association between having poor cognition and lower handgrip muscle strength through a 7-year cross-sectional study, with community-dwelling older adults. Accordingly, in our study, upper body strength showed itself as positively associated with cognitive function (Fig. 1) in institutionalized older adults with dementia.

Deterioration of functional capacity, in general, represents an increased risk of frailty, loss of independence and physical disability $[35,36]$. In agreement with the literature, our data have shown that upper and lower body strength was significantly associated with functional capacity and QoL (Figs. 2, 3, 4, 5). Although the functional capacity and QoL are generally considered to be directly connected, in the present study we observed that only lower body strength, in the multivariate model, was still positively associated with QoL from the caregivers' perspective (Table 2). Since lower body strength is crucial to perform daily tasks (e.g. bathing, transferring or dressing) with a higher degree of autonomy, we might speculate that the caregivers related it with a better QoL.

\section{Flexibility}

Articular mobility may reduce across the age span [36] and can compromise functional capacity, as well as contributing towards the loss of autonomy and independence $[37,38]$. This could be even more problematic in 
institutionalized older adults with dementia. The range of motion of the upper limb joints has been considered imperative for the ability to perform ADLs [38]. Additionally, decreased flexibility of the lower limbs has been associated with the risk of injuries and falls and changes in the gait pattern [37]. Although the present study failed to find an association between lower body flexibility and functional capacity, a significant association between upper body flexibility with cognition and functional capacity was found in this frail population. This can suggest that some ADL (such as bathing and dressing) performed autonomously, requires upper body flexibility and may be cognitively challenging for people with dementia. Unexpectedly, no significant results were observed between flexibility and QoL.

\section{Agility/dynamic balance}

Our results showed that better agility/dynamic balance scores were positively associated with general cognition. Older adults that have a better agility/dynamic balance also have more opportunities for physical practice, more autonomy and better environmental interaction, which could be important for maintaining their cognitive function. Evidence shows that cognition plays a key role in the regulation and control of mobility [39]. This motor behaviour involves dissociable neural systems which control gait initiation, planning and execution and the adaptation of these movements to meet motivational and environmental demands [40]. Cross-sectional studies have shown that the level of cognition is related to mobility, in community-dwelling older adults [41-44]. Agility/dynamic balance is inversely associated with the risk of falls [37, 45] being one of the main geriatric problems that significantly increase the risk of hospitalization and institutionalization [46], consequently decreasing their QoL. In our study, agility/dynamic balance scores were positively associated with participants' perception, caregivers' perception and global QoL. After a multivariate linear regression, agility/dynamic balance was still positively associated with global QoL. These results corroborate the literature that had already established the link between mobility and QoL, in older adults without dementia [47]. Furthermore, Davis et al. [48] pointed to agility/dynamic balance as a predictor of health-related QoL in community-dwelling older adults.

\section{Aerobic endurance}

Aerobic endurance is the most frequently studied component of physical fitness in older adults with dementia, being considered a predictor of cognitive performance
[49], in healthy older adults [50] and the most effective type of exercise to improve cognition in older adults with dementia [13]. Our study results show a positive association between aerobic endurance with cognition, corroborating other studies' results that have shown that higher levels of aerobic fitness are related to better brain health [51-53] and cognition [54], preserving critical brain areas in cognitively healthy older adults [51] and persons with $\mathrm{AD}$ [55], as well as reduced brain atrophy in those with early-stage AD [56]. Multiple mechanisms may account for associations between aerobic endurance and cognitive function. Higher aerobic fitness is linked with upregulation of neurotrophins (e.g. the brain-derived neurotrophic factor, BDNF), neurovascular plasticity (primarily via angiogenesis) and neurogenesis contributing to better cerebral health $[57,58]$. BDNF levels (which contribute to growth regulation, maintenance and survival of neurons) can be increased by aerobic endurance training [57, $59,60]$. In our study, aerobic endurance is also associated with functional capacity. After a multivariate linear regression, aerobic endurance is still positively associated with functional capacity. These results are according to the literature [61-63] that have shown aerobic endurance capacity as a physical fitness component that is directly and independently associated with the ability to perform ADL independently.

\section{Conclusion}

In summary, we believe that our findings contribute to $\underline{a}$ better understanding of the impact of different physical fitness components on cognition, functional capacity and QoL in institutionalized older adults with dementia. Although aerobic endurance stands out as the key factor of physical fitness in association with cognitive function, functional capacity and QoL, the results of this study suggest that the contribution of every component of physical fitness is singular and irreplaceable. Thus, strategies to attenuate the decline of different physical fitness components, such as the implementation of multicomponent exercise interventions (combined aerobic exercise and flexibility or strength and agility/balance training programs), may contribute to preserve cognitive function, functional capacity and to maintain QoL, in this particular population.

Acknowledgements The authors would like to thank all participants, caregivers and institutions for participating in this study

Author contribution AS planned and performed the evaluations, analysed data, interpreted results and drafted the manuscript. IMA analysed data, interpreted results and edited and revised the manuscript. ASeabra analysed data, interpreted results and revised the manuscript. 
JM edited and revised the manuscript. EM edited and revised the manuscript. JC edited and revised the manuscript.

Funding This work was supported by the Portuguese Foundation of Science and Technology (FTC) Grants as follows: SFRH/ BD/90013/2012 to AS; POCI-01-0145-FEDER-031808 to JC and FCT/UID/00617/2019 to CIAFEL.

Data availability The data that support the findings of this study are available from the corresponding author upon reasonable request.

\section{Compliance with ethical standards}

Conflict of interest The authors declare that they have no conflict of interest.

Ethical approval All procedures performed in studies involving human participants were in accordance with the ethical standards of the institutional and/or national research committee and with the 1964 Helsinki Declaration and its later amendments or comparable ethical standards. The study protocol was accepted by the Ethical Commission of the Faculty of Sports of the University of Porto (Ref CEFADE02.2014).

Informed consent Informed consent was obtained from the patients or substitute decision-makers if the patients did not have the capacity to consent.

Open Access This article is licensed under a Creative Commons Attribution 4.0 International License, which permits use, sharing, adaptation, distribution and reproduction in any medium or format, as long as you give appropriate credit to the original author(s) and the source, provide a link to the Creative Commons licence, and indicate if changes were made. The images or other third party material in this article are included in the article's Creative Commons licence, unless indicated otherwise in a credit line to the material. If material is not included in the article's Creative Commons licence and your intended use is not permitted by statutory regulation or exceeds the permitted use, you will need to obtain permission directly from the copyright holder. To view a copy of this licence, visit http://creativecommons.org/licenses/by/4.0/.

\section{References}

1. Dua T et al (2017) World Health Organization's Global Action Plan on the Response to Dementia 2017-2025. J Alzheimer Assoc 13:1450-1451

2. Alzheimer's Disease International (2015) World Alzheimer Report 2015: the global impact of dementia: an analysis of prevalence, incidence, cost and trends.

3. Shah $\mathrm{H}$ et al (2016) Research priorities to reduce the global burden of dementia by 2025. Lancet Neurol 15:1285-1294

4. Caspersen CJ, Powell KE, Christenson GM (1985) Physical activity, exercise, and physical fitness: definitions and distinctions for health-related research. Public Health Rep 100:126

5. Hesseberg K et al (2016) Physical fitness in older people with mild cognitive impairment and dementia. J Aging Phys Act 24:92-100

6. Sun F, Norman IJ, While AE (2013) Physical activity in older people: a systematic review. BMC Public Health 13:1

7. Rikli RE, Jones CJ (2013) Senior fitness test manual, 2nd edn. Human Kinetics, Champaign, IL

8. Wang T-J (2004) Concept analysis of functional status. Int J Nurs Stud 41:457-462
9. Verbrugge LM, Jette AM (1994) The disablement process. Soc Sci Med 38:1-14

10. Anderiesen $\mathrm{H}$ et al (2014) A systematic review-physical activity in dementia: the influence of the nursing home environment. Appl Ergon 45:1678-1686

11. Liu R et al (2012) Cardiorespiratory fitness as a predictor of dementia mortality in men and women. Med Sci Sports Exerc 44:253-259

12. Hamer M, Chida Y (2009) Physical activity and risk of neurodegenerative disease: a systematic review of prospective evidence. Psychol Med 39:3-11

13. Forbes D et al (2015) Exercise programs for people with dementia. Cochrane Database Syst Rev. https://doi.org/10.1002/14651858. CD006489.pub3

14. Karssemeijer EGA et al (2017) Positive effects of combined cognitive and physical exercise training on cognitive function in older adults with mild cognitive impairment or dementia: a meta-analysis. Ageing Res Rev 40:75-83

15. Northey JM et al (2018) Exercise interventions for cognitive function in adults older than 50: a systematic review with metaanalysis. Br J Sports Med 52:154-160

16. Ferrucci $L$ et al (2004) Designing randomized, controlled trials aimed at preventing or delaying functional decline and disability in frail, older persons: a consensus report. J Am Geriatr Soc 52:625-634

17. Conde-Sala JL et al (2014) Discrepancies regarding the quality of life of patients with Alzheimer's disease: a three-year longitudinal study. J Alzheimers Dis 39:511-525

18. World Medical Association (2009) Declaration of Helsinki. Ethical principles for medical research involving human subjects. J Indian Med Assoc 107(6):403-405

19. Hesseberg K, Bentzen H, Bergland A (2015) Reliability of the senior fitness test in community-dwelling older people with cognitive impairment. Physiother Res Int 20:37-44

20. Folstein MF, Folstein SE, McHugh PR (1975) "Mini-mental state": a practical method for grading the cognitive state of patients for the clinician. J Psychiatr Res 12:189-198

21. Morgado J et al (2009) Novos valores normativos do mini-mental state examination. Sinapse 9:10-16

22. Sequeira C (2007) Cuidar de idosos dependentes. Quarteto, Coimbra

23. Katz $\mathrm{S}$ et al (1963) Studies of illness in the aged the index of Adl: a standardized measure of biological and psychosocial function. JAMA 185:914-919

24. Logsdon RG et al (2002) Assessing quality of life in older adults with cognitive impairment. Psychosom Med 64:510-519

25. Manini TM, Pahor M (2009) Physical activity and maintaining physical function in older adults. Br J Sports Med 43:28-31

26. Chou CH, Hwang CL, Wu YT (2012) Effect of exercise on physical function, daily living activities, and quality of life in the frail older adults: a meta-analysis. Arch Phys Med Rehabil 93:237-244

27. Tolea MI, Galvin JE (2015) Sarcopenia and impairment in cognitive and physical performance. Clin Interv Aging 10:663-671

28. Gillette-Guyonnet $S$ et al (2000) Determination of appendicular muscle mass by dual energy X-ray absorptiometry method in women with sarcopenia and Alzheimer's disease. J Nutr Health Aging 4:165-169

29. Auyeung TW et al (2008) Functional decline in cognitive impairment-the relationship between physical and cognitive function. Neuroepidemiology 31:167-173

30. Perkisas S, Vandewoude M (2016) Where frailty meets diabetes. Diabetes Metab Res Rev 32:261-267

31. White H, Pieper C, Schmader K (1998) The association of weight change in Alzheimer's disease with severity of disease and mortality: a longitudinal analysis. J Am Geriatr Soc 46:1223-1227 
32. Burns JM et al (2010) Reduced lean mass in early Alzheimer disease and its association with brain atrophy. Arch Neurol 67:428-433

33. Nourhashémi F et al (2002) Is there a relationship between fat-free soft tissue mass and low cognitive function? Results from a study of 7105 women. J Am Geriatr Soc 50:1796-1801

34. Raji MA et al (2005) Cognitive status, muscle strength, and subsequent disability in older Mexican Americans. J Am Geriatr Soc 53:1462-1468

35. Manty M et al (2014) Indoor mobility-related fatigue and muscle strength in nonagenarians: a prospective longitudinal study. Aging Clin Exp Res 26:39-46

36. Stathokostas L et al (2013) Flexibility of older adults aged 55-86 years and the influence of physical activity. J Aging Res 2013:743843

37. ACSM, Ehrman JK, Liguori G, Magal M (2018) ACSM's guidelines for exercise testing and prescription, 10th edn. Wolters Kluwer, Philadelphia

38. Gates DH et al (2016) Range of motion requirements for upper-limb activities of daily living. Am J Occup Ther 70:7001350010p1-7001350010p10

39. Montero-Odasso $\mathrm{M}$ et al (2015) Mobility and cognition in seniors. Report from the Institute of Aging (CIHR) mobility and cognition workshop. Can Geriatr J 18:159-167

40. Rossignol S, Dubuc R, Gossard JP (2006) Dynamic sensorimotor interactions in locomotion. Physiol Rev 86:89-154

41. Ble A et al (2005) Executive function correlates with walking speed in older persons: the InCHIANTI study. J Am Geriatr Soc 53:410-415

42. de Oliveira Silva F et al (2019) Three months of multimodal training contributes to mobility and executive function in elderly individuals with mild cognitive impairment, but not in those with Alzheimer's disease: a randomized controlled trial. Maturitas 126:28-33

43. Soumare A et al (2009) A cross-sectional and longitudinal study of the relationship between walking speed and cognitive function in community-dwelling elderly people. J Gerontol A Biol Sci Med Sci 64:1058-1065

44. Buchman AS et al (2011) Cognitive function is associated with the development of mobility impairments in community-dwelling elders. Am J Geriatr Psychiatry 19:571-580

45. Shaw FE et al (2003) Multifactorial intervention after a fall in older people with cognitive impairment and dementia presenting to the accident and emergency department: randomised controlled trial. BMJ 326:73

46. Meuleners LB et al (2016) Risk factors for recurrent injurious falls that require hospitalization for older adults with dementia: a population based study. BMC Neurol 16:188

47. Fagerstrom C, Borglin G (2010) Mobility, functional ability and health-related quality of life among people of 60 years or older. Aging Clin Exp Res 22:387-394

48. Davis JC et al (2015) Mobility predicts change in older adults' health-related quality of life: evidence from a Vancouver falls prevention prospective cohort study. Health Qual Life Outcomes 13:101

49. Jonasson LS et al (2016) Aerobic exercise intervention, cognitive performance, and brain structure: results from the physical influences on brain in Aging (PHIBRA) Study. Front Aging Neurosci $8: 336$

50. Barnes DE et al (2003) A longitudinal study of cardiorespiratory fitness and cognitive function in healthy older adults. J Am Geriatr Soc 51:459-465

51. Kramer AF, Colcombe S (2018) Fitness effects on the cognitive function of older adults: a meta-analytic study-revisited. Perspect Psychol Sci 13:213-217

52. Gordon BA et al (2008) Neuroanatomical correlates of aging, cardiopulmonary fitness level, and education. Psychophysiology $45: 825-838$

53. Johnson NF et al (2012) Cardiorespiratory fitness is positively correlated with cerebral white matter integrity in healthy seniors. Neuroimage 59:1514-1523

54. Baker LD et al (2010) Aerobic exercise improves cognition for older adults with glucose intolerance, a risk factor for Alzheimer's disease. J Alzheimers Dis 22:569-579

55. Honea RA et al (2009) Cardiorespiratory fitness and preserved medial temporal lobe volume in Alzheimer disease. Alzheimer Dis Assoc Disord 23:188-197

56. Burns JM et al (2008) Cardiorespiratory fitness and brain atrophy in early Alzheimer disease. Neurology 71:210-216

57. Wang R, Holsinger RMD (2018) Exercise-induced brain-derived neurotrophic factor expression: Therapeutic implications for Alzheimer's dementia. Ageing Res Rev 48:109-121

58. Churchill JD et al (2002) Exercise, experience and the aging brain. Neurobiol Aging 23:941-955

59. Rasmussen $P$ et al (2009) Evidence for a release of brain-derived neurotrophic factor from the brain during exercise. Exp Physiol 94:1062-1069

60. Seifert $\mathrm{T}$ et al (2010) Endurance training enhances BDNF release from the human brain. Am J Physiol Regul Integr Comp Physiol 298:R372-R377

61. Morey MC, Pieper CF, Cornoni-Huntley J (1998) Physical fitness and functional limitations in community-dwelling older adults. Med Sci Sports Exerc 30:715-723

62. Bronas UG et al (2017) Determination of aerobic capacity via cycle ergometer exercise testing in Alzheimer's disease. Am J Alzheimers Dis Other Demenc 32:500-508

63. Alosco ML et al (2012) The 2-minute step test is independently associated with cognitive function in older adults with heart failure. Aging Clin Exp Res 24:468-474

Publisher's Note Springer Nature remains neutral with regard to jurisdictional claims in published maps and institutional affiliations. 